\title{
Active galaxy 4U 1344-60: did the relativistic line disappear?
}

\author{
J. Svoboda ${ }^{1}$, S. Bianchi ${ }^{2}$, M. Guainazzi ${ }^{1}$, G. Matt ${ }^{2}$, E. Piconcelli ${ }^{1,3}$, V. Karas ${ }^{4}$, and M. Dovčiak ${ }^{4}$ \\ ${ }^{1}$ European Space Astronomy Centre of ESA, PO Box 78, Villanueva de la Cañada, 28691 Madrid, Spain \\ e-mail: jsvoboda@sciops.esa.int \\ 2 Università degli Studi Roma Tre, via dellaVasca Navale 84, 00146 Roma, Italy \\ 3 Osservatorio Astronomico di Roma (INAF), via Frascati 33, 00040 Monteporzio Catone (Roma), Italy \\ 4 Astronomical Institute, Academy of Sciences, Boční II 1401, 14131 Prague, Czech Republic
}

Received 27 April 2012 / Accepted 28 August 2012

\section{ABSTRACT}

\begin{abstract}
Context. X-ray bright active galactic nuclei represent a unique astrophysical laboratory for studying accretion physics around supermassive black holes.

Aims. 4U 1344-60 is a bright Seyfert galaxy which revealed relativistic reflection features in the archival XMM-Newton observation. Methods. We present the spectroscopic results of new data obtained with the Suzaku satellite and compare them with the previous $X M M-$ Newton observation.

Results. The X-ray continuum of $4 \mathrm{U} 1344-60$ can be well described by a power-law component with the photon index $\approx 1.7$ modified by a fully and a partially covering local absorbers. We measured a substantial decrease of the fraction of the partially absorbed radiation from around $45 \%$ in the XMM-Newton observation to less than $10 \%$ in the Suzaku observation while the power-law slope remains constant within uncertainties. The iron line in the Suzaku spectrum is relatively narrow, $\sigma=(0.08 \pm 0.02) \mathrm{keV}$, without any suggestion for relativistic broadening. Regarding this, we interpret the iron line in the archival XMM-Newton spectrum as a narrow line of the same width plus an additional red-shifted emission around $6.1 \mathrm{keV}$.

Conclusions. No evidence of the relativistic reflection is present in the Suzaku spectra. The detected red-shifted iron line during the XMM-Newton observation could be a temporary feature either due to locally enhanced emission or decreased ionisation in the innermost accretion flow.
\end{abstract}

Key words. galaxies: active - galaxies: Seyfert - galaxies: individual: 4U 1344-60

\section{Introduction}

Bright active galaxies provide a good opportunity to probe accretion physics in the strong gravity regime and to reveal the basic parameters of the accreting black hole (see e.g. Reynolds \& Nowak 2003). In particular, the black hole angular momentum can be constrained from the relativistic features in the spectra originating in the closest neighbourhood to the black hole. Suitable tools for this purpose are the high-throughput X-ray detectors on space missions such as XMM-Newton (Jansen et al. 2001) and Suzaku (Mitsuda et al. 2007) whose data analysis we are presenting in this paper.

A sizeable sample of active galactic nuclei (AGNs) with relativistically broadened iron lines was studied by Guainazzi et al. (2006) and later by de La Calle Pérez et al. (2010). Different samples of Seyfert galaxies observed by the XMM-Newton satellite were also examined by Nandra et al. (2007), Brenneman \& Reynolds (2009), and Bhayani \& Nandra (2011). Broad iron lines in quasars were studied by Jiménez-Bailón et al. (2005). All groups concluded that X-ray spectra of a substantial fraction of AGNs possess a relativistically broadened iron line. The fraction increases with longer-exposed observations providing better statistics for spectroscopic studies.

$4 \mathrm{U} 1344-60$, the subject of the study discussed in this paper, is a bright (about 2 millicrabs) and nearby source but it has not been intensively studied so far due to its low Galactic latitude $\left(|b|=1.5^{\circ}\right)$. Although its X-ray spectrum is heavily absorbed by the Galactic interstellar matter $\left(N_{\mathrm{H}} \approx 1 \times 10^{22} \mathrm{~cm}^{-2}\right)$ it has clearly revealed an emission excess in the broadened red wing of the iron line in a previous XMM-Newton observation (Piconcelli et al. 2006).

$4 \mathrm{U}$ 1344-60 was discovered for the first time by the X-ray satellite Uhuru. In the XMM-Newton observation of Centaurus B, 4U 1344-60 appeared at the edge of the field (Obs.ID: 0092140101). Piconcelli et al. (2006) studied the EPIC-pn spectrum together with the optical observations of the source. The optical spectrum allowed for a classification of the source as an active galaxy of an intermediate type. The cosmological redshift was derived to be $z=0.012 \pm 0.002$. Its flux, $f_{2-10 \mathrm{keV}}^{\mathrm{XMM}}=3.6 \times 10^{-11} \mathrm{erg} \mathrm{cm}^{-2} \mathrm{~s}^{-1}$, makes it one of the brightest AGN in the hard X-ray sky. The luminosity, $L_{2-10 \mathrm{keV}}^{\mathrm{XMM}}=1.5 \times$ $10^{43} \mathrm{erg} / \mathrm{s}$, is typical for Seyfert galaxies. 4U 1344-60 was observed by INTEGRAL (Bird et al. 2010) with its hard X-ray flux $f_{20-40 \mathrm{keV}}^{\text {INTEGAL }}=(3.18 \pm 0.08) \times 10^{-11} \mathrm{erg} \mathrm{cm}^{-2} \mathrm{~s}^{-1}$ and $f_{40-100 \mathrm{keV}}^{\text {INTEGRL }}=$ $(4.1 \pm 0.2) \times 10^{-11} \mathrm{erg} \mathrm{cm}^{-2} \mathrm{~s}^{-1}$. It was also detected in the Swift$B A T$ survey with $f_{15-150 \mathrm{keV}}^{\mathrm{Swift} / \mathrm{BAT}} \approx(9.0 \pm 0.3) \times 10^{-11} \mathrm{erg} \mathrm{cm}^{-2} \mathrm{~s}^{-1}$ (Cusumano et al. 2010).

Residuals in the energy range, where emission features by iron line are expected, were formerly analysed by Piconcelli et al. (2006) in the XMM-Newton observation. A model consisting of the power-law continuum plus a narrow unresolved Gaussian line gave an unacceptable fit. If the line profile was allowed to be distorted by relativistic effects the fit was significantly improved. The inner disc radius was found to be within 10 gravitational radii $\left(r_{\mathrm{g}} \equiv \frac{G M}{c^{2}}\right)$. The outer one was larger 
than $90 r_{\mathrm{g}}$ with a fixed radial emissivity index $q=2.5$. The inclination of the disk was found to be around 20 degrees. The equivalent width of the line was about $400 \mathrm{eV}$.

$4 \mathrm{U}$ 1344-60 also exhibited a complex absorption in the X-ray spectrum. Piconcelli et al. (2006) suggested the presence of a partially covering absorber to explain a remarkably flat power law describing the data. A combined analysis of the XMM-Newton and INTEGRAL observation was later done by Panessa et al. (2008) who concluded that the spectrum is affected by one fully and two partially covering absorbers with substantial column densities $\left(N_{\mathrm{H}}^{\mathrm{pc} 1} \approx 5 \times 10^{22} \mathrm{~cm}^{-2}\right.$, and $N_{\mathrm{H}}^{\mathrm{pc} 2} \approx$ $4 \times 10^{23} \mathrm{~cm}^{-2}$ ) and covering fractions around $50 \%$.

In this paper, we present new spectroscopic results of the recent observation of 4U 1344-60 obtained with the Suzaku satellite. We compare them with the archival XMM-Newton data, which we re-analysed. The paper is organised as follows. Section 2 describes the data reduction of the Suzaku XIS and HXD/PIN, and also the re-analysis of the XMM-Newton data. The basic timing and spectral properties of the Suzaku observation are presented in Sect. 3. We compare the spectral results from the Suzaku observation with the archival XMM-Newton data in Sect. 4. The achieved results are discussed in Sect. 5, and the main conclusions are drawn in Sect. 6. The timing and spectral analysis of Centaurus B is presented in the Appendix A, as well as the estimate of its contribution to the 4U 1344-60 HXD/PIN spectrum.

\section{Data reduction and analysis}

$4 \mathrm{U}$ 1344-60 was observed by the Suzaku satellite on January 2011 (obs. ID 705058010) for the total exposure of $100 \mathrm{ks}$. The observation was performed using XIS-nominal position. It was accompanied by a short $10 \mathrm{ks}$ observation pointed to the radio-galaxy Centaurus B (obs. ID 705059010), which is a neighbouring source on the X-ray sky. Due to its proximity, the HXD/PIN spectrum of $4 \mathrm{U} 1344-60$ is likely to be contaminated. The short observation of Centaurus B was performed to measure its flux and spectral properties below $10 \mathrm{keV}$ with the XIS detectors, in order to estimate its contribution to the HXD/PIN spectrum of 4U 1344-60. This observation strategy was possible only thanks to the very low short-time variability of the Centaurus B and its simple power-law shaped X-ray spectrum, as was suggested from the previous observations by the ASCA and XMM-Newton satellites (see Appendix).

We used the Heasoft package version $6.11 .1^{1}$ for the data reduction and also for the subsequent spectral and timing analysis. The data were processed standardly following the Suzaku Data Reduction Guide ${ }^{2}$ (version 4). For all XIS detectors, we combined both $3 \times 3$ and $5 \times 5$ modes to extract the event files. The source spectra of 4U 1344-60 and Centaurus B were obtained from a circle around the centre of the point spread function with the radius of 260 arcsec. We defined the background extraction region as an annulus around the source circle with the outer radius of 360 arcsec to avoid any contamination from the calibration source. We created the related response matrices and ancillary response files using the tools xisrmfgen and xissimarfgen. The HXD/PIN spectra were reduced with the tool hxdpinxbpi. The tuned background files were used for modelling the non $\mathrm{X}$-ray background. The standard method was used to account for cosmic X-ray background as described in the Suzaku Data Reduction Guide.

\footnotetext{
1 http://heasarc.nasa.gov/lheasoft/

2 http://heasarc.nasa.gov/docs/suzaku/analysis/abc/
}

Cross-normalisation between the Suzaku detectors were fixed to 1 for XIS 0 , free for XIS 1 and XIS 3, and fixed to 1.16 for $\mathrm{HXD} / \mathrm{PIN}$. Fits were performed in the $1-10 \mathrm{keV}$ and $15-60 \mathrm{keV}$ energy ranges for the XIS and HXD/PIN, respectively. The data below $1 \mathrm{keV}$ were not included due to large Galactic absorption. The XIS 1 data in the energy range 1.6-2.3 keV were ignored due to calibration uncertainties. We used C-statistics (Cash 1979) for fitting the (unbinned) data, which employs the appropriate Poisson distribution of count fluctuations. However, we express the goodness of the fit also with the more familiar $\chi^{2}$ statistics. Only for this purpose, we binned the spectra to contain at least 30 counts per bin because the Gaussian smooth distribution of count fluctuations is good only for a sufficient number of counts per bin (see e.g. Nousek \& Shue 1989, for comparison of C- and $\chi^{2}$-statistics). The quoted errors in the text correspond to a $90 \%$ confidence level for one interesting parameter.

Because our spectral results with the Suzaku observation differ from that reported by Piconcelli et al. (2006) we also reanalysed the archival XMM-Newton data and performed a simultaneous fit to both data sets. We used the recent version of the SAS software (11.0.0) $)^{3}$. 4U 1344-60 was observed with XMM-Newton in August 2001 (obs. ID 0092140101) for the total exposure of $37 \mathrm{ks}$. The observation was aimed to Centaurus B. $4 \mathrm{U} 1344-60$ is well detected in the EPIC-pn field-of-view while it is on the edge of the EPIC-mos1 and outside of the EPIC-mos2 field-of-view due to a relatively large offset $(\approx 14 \mathrm{arcmin})$. Therefore, we used only the EPIC-pn spectra.

The data were standardly screened - only patterns $0-4$ were used, high background flaring events were ignored (background rate $>0.4 \mathrm{cts} \mathrm{s}^{-1}$ ). The source was defined as an ellipse (in the sky $x y$-coordinates: 18 800.5, $12880.5,1946.4,909.7,327)$, which better corresponds to the shape of the point spread function due to its offset position (Read et al. 2011). The background extraction region was defined from a nearby circle in the sourcefree region of the chip. The results of our re-analysis of the XMM-Newton EPIC-pn spectrum are consistent with the results by Piconcelli et al. (2006).

\section{Suzaku view of $4 \mathrm{U} 1344-60$}

The time-averaged flux of 4U 1344-60 observed by Suzaku is similar to the flux measured by the XMM-Newton satellite, i.e. $f_{2-10 \mathrm{keV}} \approx 4 \times 10^{-11} \mathrm{erg} \mathrm{cm}^{-2} \mathrm{~s}^{-1}$, or the luminosity $L_{2-10 \mathrm{keV}} \approx$ $1.2 \times 10^{43} \mathrm{erg} \mathrm{s}^{-1}$, respectively. During the observation the brightness of the source increased by a factor of 2 - see Fig. 1 where background subtracted light curves of all Suzaku detectors are shown. However, the spectral shape did not change significantly, as it is suggested by the almost constant hardness ratio defined as a ratio of $0.5-2 \mathrm{keV}$ to $2-10 \mathrm{keV}$ spectra (see Fig. 2).

The time averaged spectra of XIS 0 , XIS 1, XIS 3, and HXD/PIN detectors are shown in Fig. 3. The HXD/PIN spectrum of $4 \mathrm{U} 1344-60$ is background-dominated with the $27 \%$ of the flux due to the source. This value is well above the background systematic error (around 3-5\%, see e.g. Suzaku-Memo2008-03). The contamination of the 4U 1344-60 HXD/PIN spectrum by Centaurus B is negligible (see Sect. 6 in Appendix A). The $15-60 \mathrm{keV}$ flux of $4 \mathrm{U} 1344-60$ is approximately $8 \times$ $10^{-11} \mathrm{erg} \mathrm{cm}^{-2} \mathrm{~s}^{-1}$ while the estimated flux from the XIS spectra of Cen B is only $8 \times 10^{-12} \mathrm{erg} \mathrm{cm}^{-2} \mathrm{~s}^{-1}$, i.e. one order of magnitude lower.

\footnotetext{
3 http://xmm.esa.int/sas/
} 


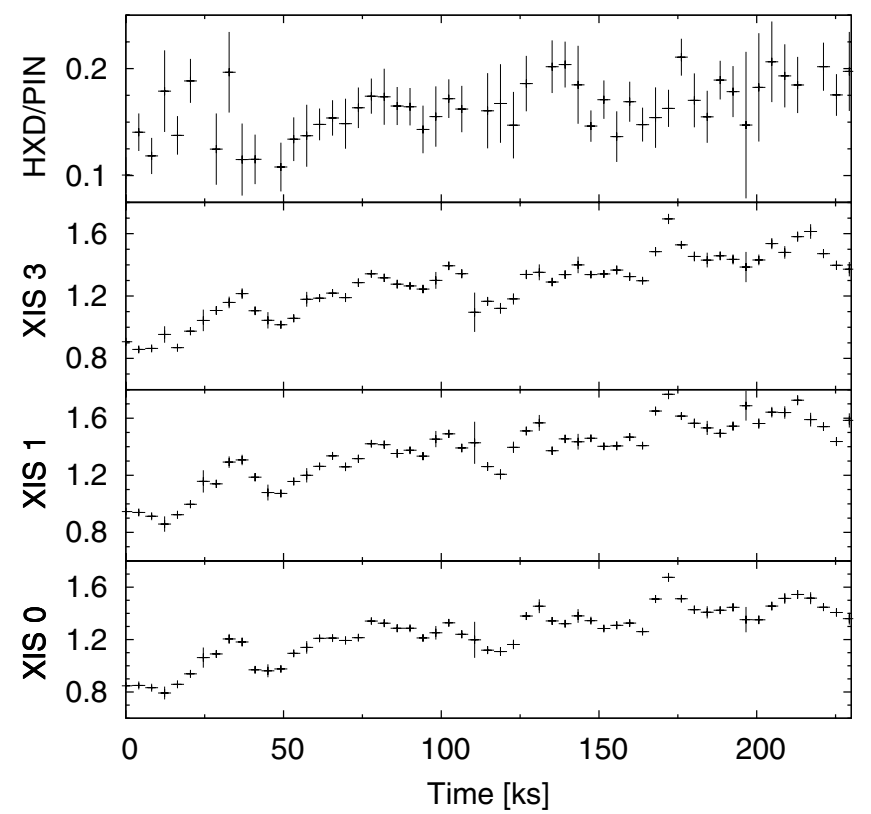

Fig. 1. XIS 0, XIS 1, XIS 3, and HXD/PIN (all background subtracted) light curves in the $0.5-10 \mathrm{keV}$, or $15-60 \mathrm{keV}$ energy range, respectively. The time bin size is $4096 \mathrm{~s}$.

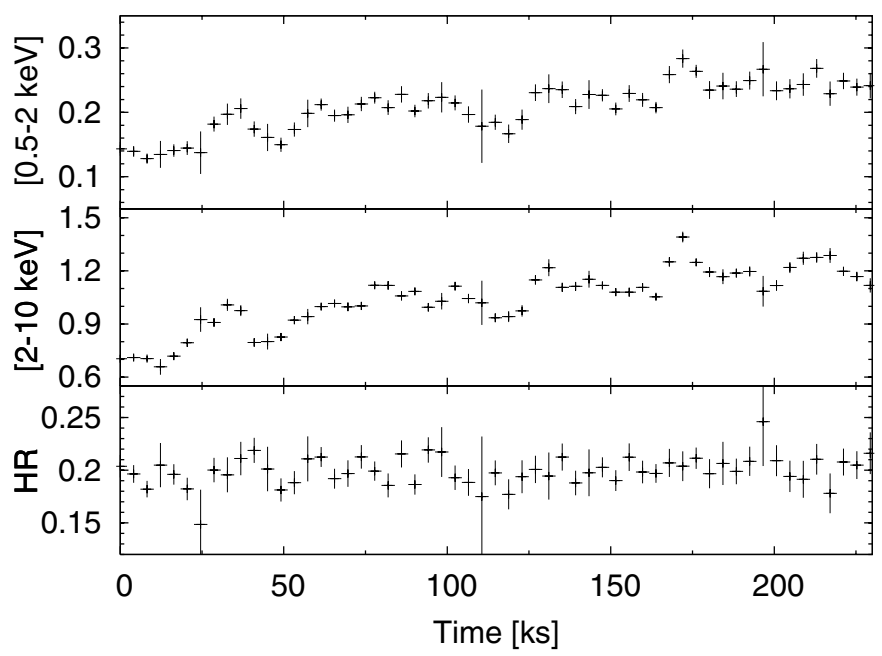

Fig. 2. XIS 0 (background subtracted) light curves in different spectral bands: $0.5-2 \mathrm{keV}$ (upper panel), and $2-10 \mathrm{keV}$ (middle panel). Their ratio is plotted in the bottom panel. The time bin size is $4096 \mathrm{~s}$.

The underlying model in Fig. 3 is an absorbed power-law with the photon index $\Gamma \approx 1.7$. The твABs model (Wilms et al. 2000) was used for the photoelectric absorption. We fixed the column density of the absorption due to inter-stellar material in our Galaxy to the value $N_{\mathrm{H}}=1.06 \times 10^{22} \mathrm{~cm}^{-2}$ (Kalberla et al. 2005). The survey by Dickey \& Lockman (1990) gives a consistent value $\left(N_{\mathrm{H}}=1.05 \times 10^{22} \mathrm{~cm}^{-2}\right)$. An additional absorption of comparable column density was required. We associated it to the host galaxy, although, regarding the very low Galactic latitude of $4 \mathrm{U}$ 1344-60, some fraction of it might be due to local variations in our Galaxy. The column density of the additional absorber was found to be $N_{\mathrm{H}}=0.93 \pm 0.02 \times 10^{22} \mathrm{~cm}^{-2}$ with the ZTBABS model.

The only clear residuals from this simple model are around $6.4 \mathrm{keV}$, and can be attributed to the iron fluorescent line. We added a Gaussian line to the model with the centroid energy and width as free parameters. The fit is then characterised by

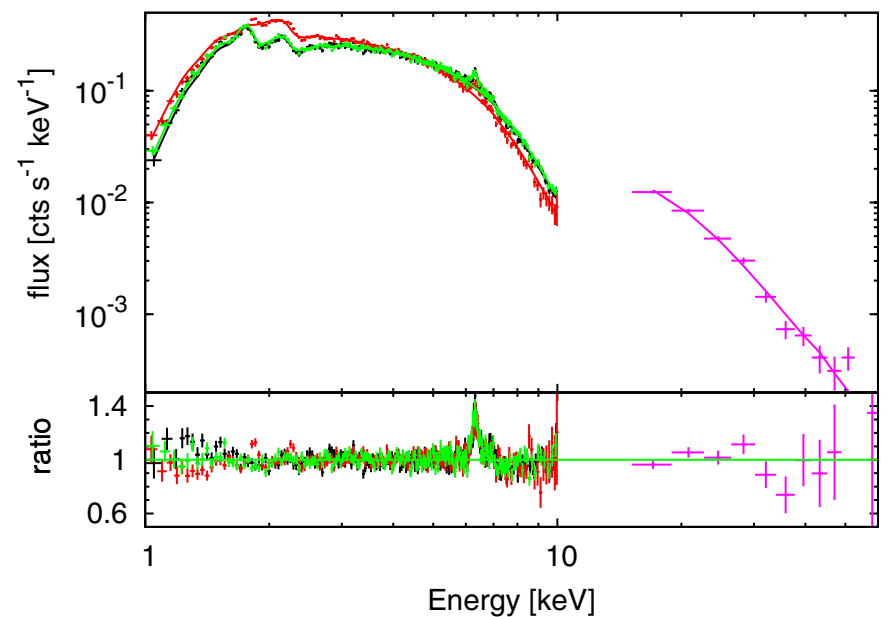

Fig. 3. XIS 0 (black), XIS 1 (red), XIS 3 (green), and HXD/PIN (magenta) spectrum. The underlying model is an absorbed power law with $\Gamma \approx 1.7$. The spectrum is binned for plotting purposes only.

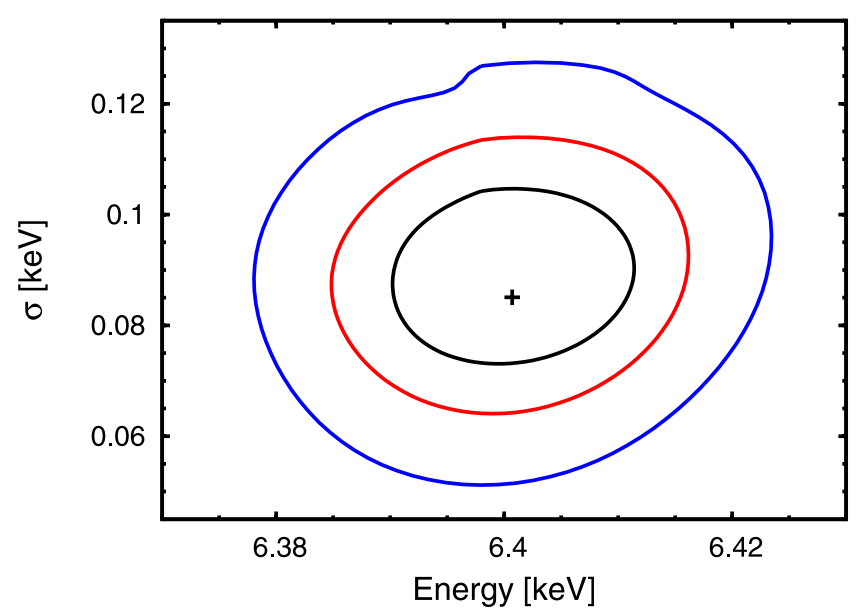

Fig. 4. Contour plot of the rest energy of the iron $\mathrm{K} \alpha$ line and its width as measured with Suzaku. The individual lines correspond to 68, 90, and $99 \%$ confidence levels. The best-fit values are indicated by a small cross inside the contours.

the C-value 7615 with 7312 degrees of freedom $(v)$, or $\chi_{v}^{2}=$ $5612 / 5452 \approx 1.03$, respectively. The equivalent width of the line is $96_{-8}^{+14} \mathrm{eV}$. The energy of the line was found to be $E=$ $6.40 \pm 0.03 \mathrm{keV}$ and its width $\sigma=0.085_{-0.015}^{+0.025} \mathrm{keV}$ (see Fig. 4).

The corresponding FWHM velocity is $\approx 8800 \mathrm{~km} \mathrm{~s}^{-1}$, which suggests an origin in the outermost regions of an accretion disc or in a broad line region (Peterson 2006). The optical measurements of a broad component of $\mathrm{H}_{\alpha}$ by Piconcelli et al. (2006) gives FWHM velocity twice smaller $\left(\approx 4400 \mathrm{~km} \mathrm{~s}^{-1}\right)$ implying that the iron line must originate closer to the black hole than the optical broad line region. However, compared to the $X M M-N e w t o n$ observation with $E \approx 6.2 \mathrm{keV}$, and $\sigma \approx 0.3 \mathrm{keV}$ (Piconcelli et al. 2006) the Suzaku iron line profile is much narrower. Therefore, we perform a simultaneous fit of both observations in the next section.

\section{Comparison with the XMM-Newton observation}

\subsection{Evidence of long-term spectral variability}

Although the time-averaged observed flux did not change significantly between the XMM-Newton and Suzaku observations the 


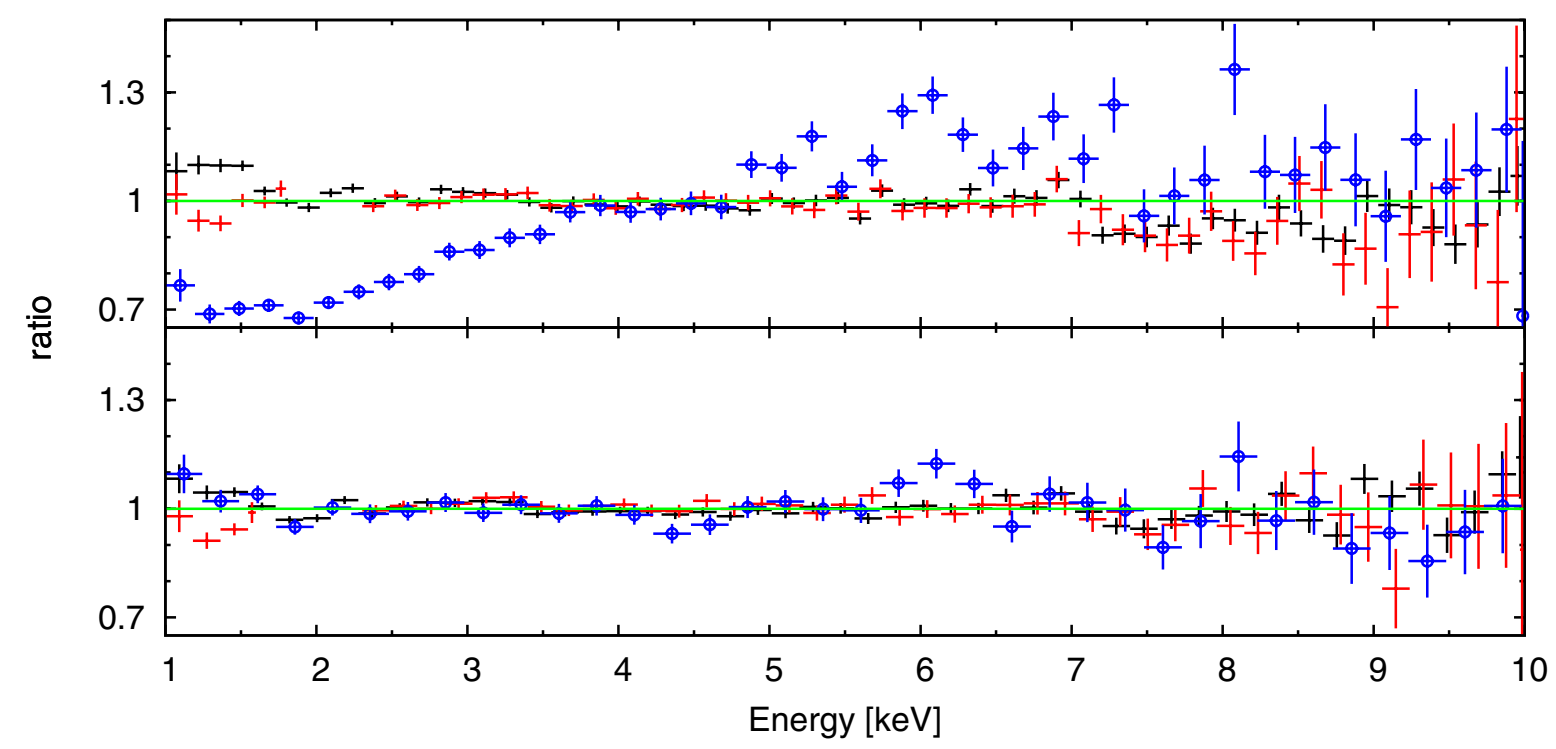

Fig. 5. Comparison of Suzaku and XMM-Newton data residuals. Up: data residuals from the identical underlying model consisting of an absorbed power law and a Gaussian line. Bottom: data residuals from the final model containing an additional absorber with different covering fraction and slightly different slope of the power law for the XMM-Newton and the Suzaku observation. The front-illuminated spectra of XIS 0 and XIS 3 are jointly shown by black data points, the back-illuminated XIS 1 are red, and the EPIC-pn data are blue and emphasised by small circles. The data are binned for plotting purposes only.

spectral shape is different. The simple power-law model with a Gaussian line applied to both data sets with the same parameters as from the Suzaku fit (besides the normalisation factors) does not provide an acceptable fit to the EPIC-pn spectrum with the total $C / v=10476 / 9112$, or $\chi_{v}^{2}=7340 / 6219 \approx 1.2$, respectively. Figure 5 (upper panel) clearly shows that either the photon index of the power law or the properties of the absorber changed between these two observations.

\subsection{Model with a variable power-law slope}

First, we allowed the column density of the local absorber to vary. The fit improved to $C / v=9877 / 9113$, or $\chi_{v}^{2}=$ $6741 / 6220 \approx 1.08$, respectively. The value of the column density of the local absorber was found to be $N_{\mathrm{H}, \mathrm{XMM}}=(1.5 \pm 0.1) \times$ $10^{22} \mathrm{~cm}^{-2}$. A significantly better fit $\left(C / v=9554 / 9111\right.$, or $\chi_{v}^{2}=$ $6468 / 6218 \approx 1.04$, respectively) was found when we also relaxed the power-law photon index. New values are $\Gamma_{\mathrm{XMM}}=$ $1.24 \pm 0.04$ and $N_{\mathrm{H}, \mathrm{XMM}}=(0.85 \pm 0.06) \times 10^{22} \mathrm{~cm}^{-2}$. The fit goodness further improved by $\Delta C \approx 11$ when, in addition, the iron line parameters were unbound. The resulting fit parameters are summarised in Table 1 in the column labelled "flat power-law model".

The energy of the iron line is significantly red-shifted towards its rest energy and broadened, consistently with the findings by Piconcelli et al. (2006). We replaced the Gaussian line by the relativistic iron line model KYRLINE (Dovčiak et al. 2004b). The resulting fit improved by $\Delta C \approx 22$ with respect to the model with a broad Gaussian line, suggesting significant asymmetry of the line profile. The angular momentum of the black hole is preferred to be high, i.e. the disc extends very close to the black hole. The inclination angle was found to be $i=(22 \pm 7)^{\circ}$. We assumed the standard radial emissivity profile (i.e. reflection flux $\propto r^{-3}$ ) and isotropic angular emissivity. Figure 6 shows the confidence levels of the spin and the inclination angle.

Although this model can satisfactorily explain the $X M M-N e w t o n$ spectrum, the narrow line from the Suzaku spectrum suggests that the reflection originates much further

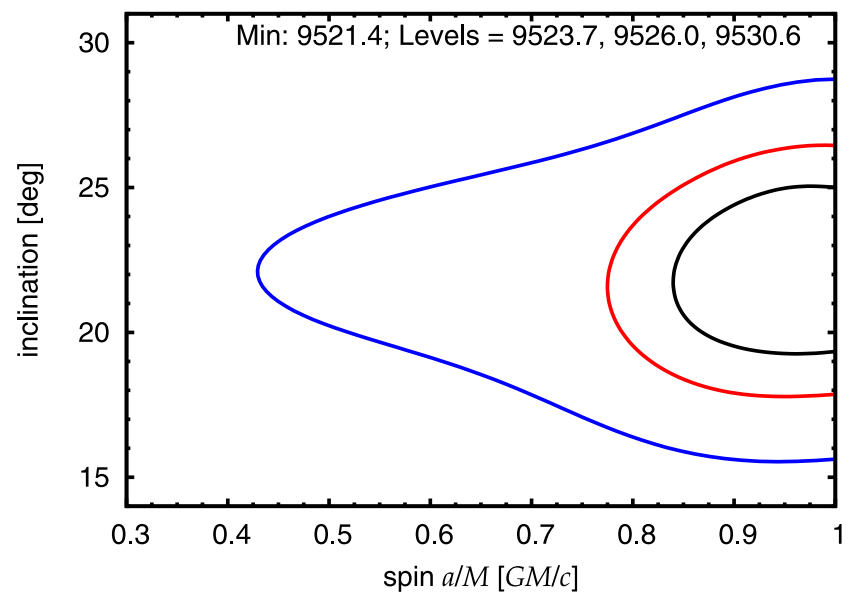

Fig. 6. Contour plot of the spin and the inclination angle measured by the relativistic iron $\mathrm{K} \alpha$ line in the flat power-law model. The individual lines correspond to 68,90 , and $99 \%$ confidence levels.

from the central black hole. A major part of the iron-line profile can be indeed explained by the same narrow line as seen in the Suzaku spectrum (see Sect. 5.2 for more discussion). Therefore, we proposed an alternative model explaining the spectral change between the XMM-Newton and Suzaku observation.

\subsection{Model with a complex absorber's variability}

Piconcelli et al. (2006) suggested that a partially covering absorption might be responsible for measuring the flat spectral shape in the XMM-Newton observation. Employing the model with more complex absorption did not improve the statistical goodness of the fit, but yielded a photon index more similar to the values measured in Seyfert galaxies (see e.g. Bianchi et al. 2009). Therefore, we also included the partially covering absorption in our model, first only to the XMM-Newton data. We bound the iron-line parameters between the Suzaku and XMM-Newton 
Table 1. Spectral analysis of $4 \mathrm{U} 1344-60$ - the model parameters.

\begin{tabular}{|c|c|c|c|c|c|c|c|c|c|}
\hline \multirow[t]{2}{*}{ Model component } & \multirow[t]{2}{*}{ Parameter } & \multicolumn{2}{|c|}{ Flat power-law model } & \multicolumn{2}{|c|}{ "Part. cov." model } & \multicolumn{2}{|c|}{ Complex model "fix" } & \multicolumn{2}{|c|}{ Complex model "free" } \\
\hline & & $X M M$ & Suzaku & $X M M$ & Suzaku & $X M M$ & Suzaku & $X M M$ & Suzaku \\
\hline local absorption & $n_{\mathrm{H}, \text { fully }}\left[10^{22} \mathrm{~cm}^{-2}\right]$ & $0.86_{-0.06}^{+0.07}$ & $0.93 \pm 0.02$ & \multicolumn{2}{|c|}{$0.92 \pm 0.02$} & \multicolumn{2}{|c|}{$0.95 \pm 0.02$} & \multicolumn{2}{|c|}{$0.98 \pm 0.02$} \\
\hline$\cdots$ & $n_{\mathrm{H}, \mathrm{part}}\left[10^{22} \mathrm{~cm}^{-2}\right]$ & \multicolumn{2}{|r|}{$\cdots$} & \multicolumn{2}{|c|}{$7.5_{-1.2}^{+1.3}$} & \multicolumn{2}{|c|}{$9.3_{-1.2}^{+1.3}$} & \multicolumn{2}{|c|}{$8.2 \pm 1.5$} \\
\hline$\cdots$ & cov. fraction [\%] & \multicolumn{2}{|r|}{$\cdots$} & $44_{-13}^{+15}$ & $6 \pm 3$ & $50 \pm 3$ & $5 \pm 3$ & $41_{-13}^{+15}$ & $10 \pm 6$ \\
\hline power law & $\Gamma$ & $1.26_{-0.02}^{+0.04}$ & $1.68_{-0.01}^{+0.02}$ & $1.66 \pm 0.09$ & $1.72 \pm 0.02$ & \multicolumn{2}{|c|}{$1.72_{-0.02}^{+0.03}$} & $1.68_{-0.09}^{+0.08}$ & $1.83 \pm 0.06$ \\
\hline reflection & $R$ & \multicolumn{2}{|r|}{$\ldots$} & \multicolumn{2}{|c|}{$\ldots$} & \multicolumn{2}{|c|}{$0.48 \pm 0.05$} & \multicolumn{2}{|c|}{$2 \pm 1$} \\
\hline$\ldots$ & $E_{\text {fold }}$ & \multicolumn{2}{|r|}{$\cdots$} & \multicolumn{2}{|c|}{$\cdots$} & \multicolumn{2}{|c|}{$100(\mathrm{f})$} & \multicolumn{2}{|c|}{$200 \pm 100$} \\
\hline$\cdots$ & $i[\mathrm{deg}]$ & \multicolumn{2}{|r|}{$\cdots$} & \multicolumn{2}{|c|}{$\cdots$} & \multicolumn{2}{|c|}{45 (f) } & \multicolumn{2}{|c|}{$85_{-6}$} \\
\hline$\cdots$ & $N_{\text {dir }}\left[10^{-3}\right]$ & $5.7_{-0.4}^{+0.3}$ & $10.9 \pm 0.2$ & $7.1 \pm 0.4$ & $11.0 \pm 0.3$ & $7.6_{-0.6}^{+0.7}$ & $11.2 \pm 0.2$ & $7.5 \pm 0.5$ & $12.2 \pm 0.6$ \\
\hline$\cdots$ & $N_{\mathrm{abs}}\left[10^{-3}\right]$ & \multicolumn{2}{|r|}{$\cdots$} & $6 \pm 2$ & $0.7_{-0.3}^{+0.4}$ & $7.6 \pm 0.3$ & $0.6 \pm 0.4$ & $5 \pm 2$ & $1.4 \pm 0.8$ \\
\hline Gaussian line & $E_{\text {rest }}[\mathrm{keV}]$ & $6.26_{-0.07}^{+0.08}$ & $6.40 \pm 0.01$ & $6.29_{-0.07}^{+0.06}$ & $6.40 \pm 0.01$ & \multicolumn{2}{|c|}{$\cdots$} & \multicolumn{2}{|c|}{$\cdots$} \\
\hline$\ldots$ & $\sigma[\mathrm{keV}]$ & $0.23_{-0.08}^{+0.09}$ & $0.08_{-0.01}^{+0.02}$ & $0.21_{-0.07}^{+0.06}$ & $0.08 \pm 0.02$ & \multicolumn{2}{|c|}{$\ldots$} & \multicolumn{2}{|c|}{$\cdots$} \\
\hline$\ldots$ & $N_{\text {line }}\left[10^{-5}\right]$ & $10 \pm 3$ & $4.7_{-0.4}^{+0.6}$ & $9 \pm 3$ & $4.7_{-0.6}^{+0.5}$ & \multicolumn{2}{|c|}{$\cdots$} & \multicolumn{2}{|c|}{$\cdots$} \\
\hline fit goodness & $C / v$ & \multicolumn{2}{|c|}{$9543 / 9110$} & \multicolumn{2}{|c|}{$9484 / 9108$} & \multicolumn{2}{|c|}{$9477 / 9114$} & 946 & /9111 \\
\hline
\end{tabular}

Notes. The common parameters for all the fits are the column density of the absorber due to interstellar matter in our Galaxy $n_{\mathrm{HISM}}=1.06 \times$ $10^{22} \mathrm{~cm}^{-2}$ (Kalberla et al. 2005), and the cosmological redshift $z=0.012 \pm 0.002$ (Piconcelli et al. 2006). Solar abundances were assumed in the reflection models. The $N_{\text {dir }}$ parameter is a normalisation factor of the direct component while $N_{\text {abs }}$ corresponds to the component affected by the partially covering absorber. They belong to the power-law, or the reflection model by PEXMON, respectively. The sign "(f)" means that the parameter was fixed to that value.

spectra for the case that the spectral curvature at around 5-6 keV could be explained by partially absorbing clouds, similarly as in spectroscopy studies of different sources by Miller et al. (2008) or Turner et al. (2011).

The photon index of the XMM-Newton spectrum, $\Gamma=1.6 \pm$ 0.1 , is indeed found to be more consistent with the Suzaku observation. The goodness of the fit is also better than in the previous case with $C / v=9499 / 9109$, or $\chi_{v}^{2}=6408 / 6216 \approx 1.03$, respectively. The column density of the fully covering absorption was found to be $N_{\mathrm{H}, \mathrm{XMM} \text {,fully }}=0.86 \pm 0.11 \times 10^{22} \mathrm{~cm}^{-2}$. The fraction of the partially absorbed power law is $44 \pm 12 \%$ with the column density $N_{\mathrm{H}, \mathrm{XMM} \text {,part }}=7.1_{-1.8}^{+2.0} \times 10^{22} \mathrm{~cm}^{-2}$.

As a next step, we included the partially covering absorption also for the Suzaku data. We simply assumed that only the fraction of the partially covering absorption changed between the times of the two observations (allowing the column densities to vary did not improve the fit significantly). The column density of the fully covering absorption was found to be $N_{\mathrm{H} \text {,fully }}=$ $0.92 \pm 0.02 \times 10^{22} \mathrm{~cm}^{-2}$, the column density of the partial covering $N_{\mathrm{H} \text {,part }}=7.5_{-1.2}^{+1.3} \times 10^{22} \mathrm{~cm}^{-2}$, and the fraction of the partially absorbed power law $44_{-13}^{+15} \%$ for the XMM-Newton and $5 \pm 3 \%$ for the Suzaku spectrum. The fit goodness is $C / v=9490 / 9110$, or $\chi_{v}^{2}=6347 / 6217 \approx 1.02$, respectively.

Piconcelli et al. (2006) rejected the model with the partially covering absorber (their "model E"), because it did not explain all the residuals at the iron-line energy band. Indeed, when we unbound the iron-line parameters, we obtained similar results as with the flat-powerlaw model. The energy of the line is found red-shifted, $E=6.3 \pm 0.1 \mathrm{keV}$, and broadened, $\sigma=0.2 \pm 0.1 \mathrm{keV}$. The equivalent width of the line is $E W_{\mathrm{SUZ}}=96_{-12}^{+10} \mathrm{eV}$ with

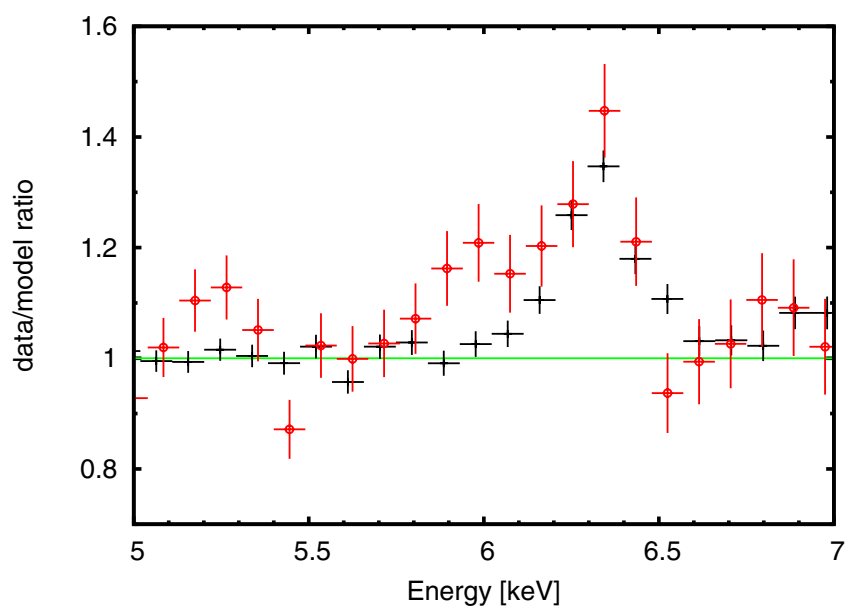

Fig. 7. Spectral residuals in the iron line energy range. The residuals are calculated against model consisting of power law with the partially covering absorber. Black data points correspond to the spectra of frontilluminated Suzaku/XIS detectors. The red ones (with small circles) correspond to EPIC-pn spectrum. The data are binned for plotting purposes only.

Suzaku, and $E W_{\mathrm{XMM}}=160_{-50}^{+60} \mathrm{eV}$ with XMM-Newton. The difference consists in the width of the line. A more detailed look on the iron-line residuals is shown in Fig. 7. The partially-covering absorber does not explain why the iron line is broader and more redshifted in the XMM-Newton spectrum. However, we consider this model further, as it is more consistent with the Suzaku measurements than the flat power-law model. 
Table 2. Flux of 4U 1344-60 measured by different instruments and predicted values by various models.

\begin{tabular}{l|c|c|c|c|c}
\hline \hline \multirow{2}{*}{ Instrument (- model) } & \multicolumn{5}{|c}{ Flux $\left[10^{-11} \mathrm{erg} \mathrm{cm}^{-2} \mathrm{~s}^{-1}\right]$} \\
\hline & $2-10 \mathrm{keV}$ (observed) & $2-10 \mathrm{keV}$ (intrinsic) & $20-40 \mathrm{keV}$ & $40-100 \mathrm{keV}$ & $20-100 \mathrm{keV}$ \\
\hline$X M M-$ flat $\Gamma$ & $4.16 \pm 0.04$ & $4.71 \pm 0.04$ & $7.7 \pm 0.1(\mathrm{p})$ & $19.0 \pm 0.2(\mathrm{p})$ & $26.7 \pm 0.3(\mathrm{p})$ \\
$X M M-$ part. cov. & $4.04 \pm 0.04$ & $5.56 \pm 0.04$ & $4.3 \pm 0.1(\mathrm{p})$ & $7.5 \pm 0.1(\mathrm{p})$ & $11.9 \pm 0.2(\mathrm{p})$ \\
SUZAKU - part. cov. & $3.83 \pm 0.01$ & $4.58 \pm 0.02$ & $3.29 \pm 0.02$ & $5.49 \pm 0.03(\mathrm{p})$ & $8.75 \pm 0.05(\mathrm{p})$ \\
SUZAKU - final & $3.83 \pm 0.01$ & $4.67 \pm 0.02$ & $3.35 \pm 0.02$ & $4.09 \pm 0.03(\mathrm{p})$ & $7.44 \pm 0.05(\mathrm{p})$ \\
INTEGRAL & $\ldots$ & $\ldots$ & $3.2 \pm 0.1^{a}$ & $4.1 \pm 0.2^{a}$ & $6.6^{b}$ \\
Swift-BAT & $\ldots$ & $\ldots$ & $2.7 \pm 0.1$ & $4.0 \pm 0.2$ & $6.7 \pm 0.3$ \\
\hline
\end{tabular}

Notes. A sign (p) means that the value was predicted by the model from lower X-ray energies. ${ }^{(a)}$ Bird et al. (2010), ${ }^{(b)}$ Panessa et al. (2008).

\subsection{More complex reflection models}

The iron line detected in the XIS spectra implies the presence of the cold reflection component in the spectrum. We used further PEXMON model (Nandra et al. 2007), which combines the reflection continuum by PEXRAV model (Magdziarz \& Zdziarski 1995) with the appropriate strength of the fluorescent lines of iron and nickel. Because the iron line detected by XIS detectors is narrow, the reflection must occur sufficiently far from the black hole and is rather unlikely to be variable between the two observations. Therefore, we simply assumed the same reflection model for both Suzaku and archival XMM-Newton data.

Neither the inclination angle nor the origin of the reflection is known in this galaxy. Piconcelli et al. (2006) determined the type of Seyfert 1.5 galaxy from the optical measurements. In terms of the AGN unification scenario by Antonucci (1993), this would correspond to an intermediate value of the inclination, i.e. $i \approx 45$ degrees. Therefore we considered two cases: first one with keeping the values of the inclination and the folding energy fixed in the fit (called as "complex fix" in Table 1) and second one with these parameters free ("complex free" in Table 1, or "final" model hereafter).

The fit goodness of the final model is characterised by $C / v=$ $9460 / 9111$, or $\chi_{v}^{2}=6316 / 6218 \approx 1.02$, respectively. The final model is plotted in Fig. 8 in the $1-10 \mathrm{keV}$ energy range. It is demonstrated there how the fraction affected by the partially covering absorber decreased between the two observations. The iron and nickel fluorescent lines as well as the iron edge are the properties of non-relativistic cold reflection.

Although the spectrum is described rather satisfactorily by the final model, the residuals at the energy range around $6 \mathrm{keV}$ still persist in the XMM-Newton spectrum. The data/model ratio is shown in the bottom panel of Fig. 5. In order to demonstrate the significance of the remaining residuals, we added a Gaussian line to the final fit. The fit improves by $\Delta C=22$ (or $\Delta \chi=23$, respectively). This is a statistically significant result as the random improvement should be of order of $\Delta C \approx 6$ for the three extra parameters added to the model. The energy of the Gaussian line was found to be $E=6.15_{-0.11}^{+0.12} \mathrm{keV}$, the width $\sigma=0.21_{-0.11}^{+0.12} \mathrm{keV}$, and normalisation factor $N_{\text {Gauss }}=5.8_{-2.1}^{+1.9} \times 10^{-5}$. The equivalent width of this feature is $90_{-40}^{+100} \mathrm{eV}$. This additional line can be attributed to a redshifted iron line emitted from inner parts of the accretion disc - see Sect. 5.2 for more discussion on this spectral feature.

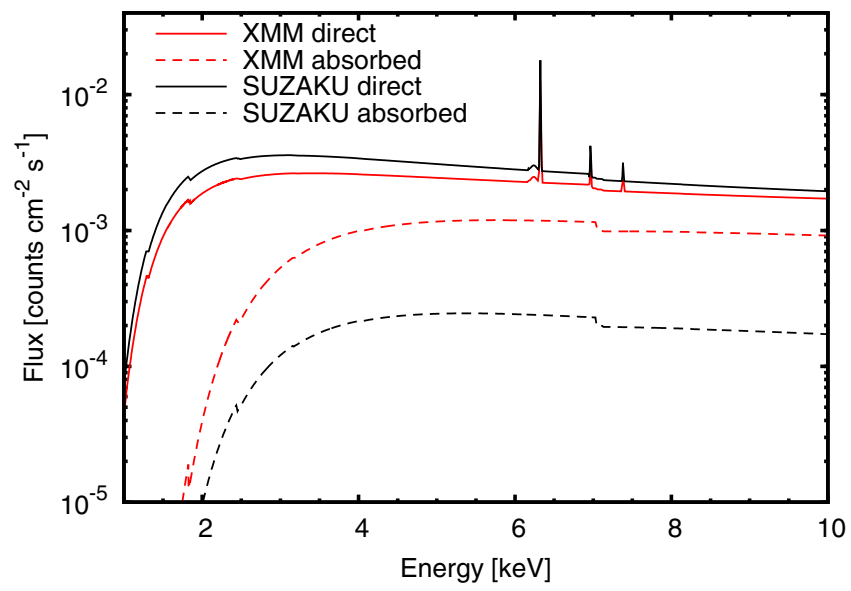

Fig. 8. Final model consisting of a partially covering absorber and cold reflection. The model is shown by red or black related to the XMM-Newton or the Suzaku observation, respectively. The components affected by the partially covering absorber are shown by dashed lines while the direct components by solid lines.

\section{Discussion}

\subsection{Presence of a partially covering absorber}

The Suzaku observation of 4U 1344-60 reveals that the spectrum can be characterised by a photon index of the power law $\Gamma \approx 1.7$, which is a typical value for Seyfert galaxies (Bianchi et al. 2009). The different slope of the XMM-Newton spectrum can be explained by a very low photon index $(\Gamma \lesssim 1.3)$ or by the presence of a partially covering absorber. A statistically better fit was obtained with the model with the partially covering absorber. Moreover, this model yields flux at high energies more consistent with the measured values by the INTEGRAL and Swift satellite, as demonstrated in Table 2.

High-energy flux predicted by different models (flat power law, model with the partially covering absorber, final model) is compared with the values measured by the INTEGRAL and Swift satellite ${ }^{4}$. Better agreement is obtained for the scenario with the partially covering absorber (best for the final model).

\footnotetext{
4 A power-law model was used to measure Swift-BAT flux from the 58month hard X-ray survey data. http://heasarc.gsfc.nasa.gov/ docs/swift/results/bs58mon/
} 
However, this cannot be regarded as a final proof owing to a possible long-term variability between the non-simultaneous observations. Moreover, a low high-energy cut-off in the flat power law would significantly decrease the predicted hard X-ray flux by this model.

An additional fully-covering absorber with $N_{\mathrm{H}} \approx 10^{22} \mathrm{~cm}^{-2}$ along our line of sight to the nucleus of $4 \mathrm{U} 1344-60$ was required by the data. It may be identified with dust lanes or star-forming regions in the innermost host galaxy disc and not associated with the AGN torus (Matt 2000). A very large contamination from the host galaxy has also been claimed by Vasudevan et al. (2010) in order to explain the infrared spectral energy distribution of $4 \mathrm{U}$ 1344-60. This scenario is consistent with the intermediate type classification (see e.g. Maiolino \& Rieke 1995), which was originally proposed for 4U 1344-60 by Piconcelli et al. (2006).

\subsection{The iron line in the XMM-Newton observation}

Model consisting of a power-law component modified by the fully and partially covering absorber and the narrow iron fluorescent line represents a good simultaneous fit to both, the Suzaku and the archival XMM-Newton spectrum, apart from the emission residuals at the moderately redshifted iron line energy band that are entirely related to the XMM-Newton spectrum. Figure 7 shows this energy band for the Suzaku-XIS and XMM-Newton spectrum in detail. Piconcelli et al. (2006) concluded from the $X M M-N e w t o n$ spectrum that the total iron line profile is most likely formed in the innermost accretion disc. However, the line profile measured by Suzaku is much narrower. Therefore, we interpreted the XMM-Newton iron line profile as the narrow component (the same as in the Suzaku spectrum) plus an additional enhanced emission from the innermost accretion disc.

Such an enhanced emission can be explained, e.g., by a temporary irradiation of a part of the accretion disc by a flare, for instance due to a magnetic reconnection in the corona (Czerny et al. 2004; Uzdensky \& Goodman 2008). With the energy $E \approx$ $6.1 \mathrm{keV}$, it is significantly redshifted when associated to the iron fluorescent line. Therefore, the probable origin is from the innermost accretion disc where the radiation is affected by strong gravitational redshift. A transient emission feature at a similar energy was reported for another Seyfert galaxy NGC 3516 (Turner et al. 2002; Bianchi et al. 2004). Much longer exposure time allowed to trace the temporal evolution of the emission and reveal possible periodicity due to an orbiting spot in the accretion disc (Iwasawa et al. 2004).

We performed several tests using KY model (Dovčiak et al. 2004b) including also non-axisymmetric models (due to the presence of a partially covering absorber). We tried to split the XMM-Newton observation into several parts and look for the change of residuals. We also generated a light curve related only to the redshifted component of the iron line and compared it with the total light curve. However, the insufficient data quality of the short XMM-Newton observation does not allow us to uniquely constrain neither the geometrical structure of the emission region nor its temporal behaviour. It was not possible to distinguish between a "ring-shaped" static emitting region and an orbiting spot. The evidence of such spectral features, however, satisfy the studies of a locally enhanced emission from parts of the accretion disc (Dovčiak et al. 2004a; Goosmann et al. 2007), and will be potentially resolved with future higher resolution X-ray instruments (see e.g. Sochora et al. 2011).

The absence of any relativistic features in the Suzaku spectrum is rather puzzling. Although the $4 \mathrm{U}$ 1344-60 observation revealed a complex absorption the $S u z a k u$ observation provided us the clearest view on the nucleus. A possible explanation for non-detection of the redshifted iron-line emission could be due to a truncation of the disc at a further radius. A truncated accretion disc was reported for several other sources (see e.g. Matt et al. 2005; Markowitz \& Reeves 2009; Svoboda et al. 2010). The physical reason for such a truncation might be due to a very low accretion rate when the accretion flow would be advection dominated and would not form a thin accretion disc (Esin et al. 1997), or due to an over-ionisation of the innermost part of the accretion disc by strong irradiation (Done et al. 2000; Bhayani \& Nandra 2011).

The change of the iron line from a relativistically broadened profile to a narrow one as a likely result of the state transition of the source, was reported for a quasar Q0056-363 (Matt et al. 2005). Different photon indices were measured between the two observations but on contrary to our case the flatter index was related to the narrow line detection. An explanation in terms of the disc truncation is therefore unlikely in the case of 4U 1344-60 since it does not suite well in a truncated disc scenario in the hard states of Galactic black hole binaries (Lubiński \& Zdziarski 2001; Fender et al. 2004). Moreover, the measured flux during the Suzaku observation is very close to that measured by XMM-Newton. The accretion rate derived from the infrared and X-ray observations is $\lambda \approx 0.03 \lambda_{\text {Edd }}$ (Vasudevan et al. 2010), which is somewhat typical value for other Seyfert galaxies as well.

Therefore, the ionisation may represent a more plausible explanation. The illuminating flux is a strongly radially dependent function, especially if the corona is very compact (Matt et al. 1993; Reynolds \& Fabian 2008; Svoboda et al. 2012). The innermost disc can be over-ionised owing to the combination of sufficiently high irradiation flux and relatively low density of the accreting material. The ionisation state could, however, locally decrease if the density is locally increased (e.g. due to a shock wave), or if some clouds partly obscure the illuminating source. The latter one could happen during the XMM-Newton observation regarding the presence of the partially covering absorber. As a result, some additional fluorescent emission was detected from the innermost part of the accretion disc.

An alternative explanation for the missing imprints of the innermost region could be due to a distant corona. Speculatively, if the scattering medium is associated with the partially covering absorber this would also explain the change between the XMM-Newton and the Suzaku observation. The decreased covering fraction would be due to an out-flowing corona and thus, less emission from the innermost part of the disc would be detected in the Suzaku observation. Smaller intrinsic X-ray flux (see Table 2) during the Suzaku observation would match well this scenario.

\subsection{Reflection models and the angle of inclination}

The reflection model PEXMON was used for a consistent description of the iron $\mathrm{K} \alpha$, the iron edge and the Compton hump. The reflection fraction $R$, the folding energy $E_{\text {fold }}$ and also the inclination angle $i$ are, however, not constrained very well and suffer from a mutual degeneration of the parameters. Moreover, the link between the Compton hump and fluorescent lines might not be necessarily fulfilled. Especially in the case of reflection from the torus a different geometry should be taken into account (Murphy \& Yaqoob 2009; Yaqoob 2012). We also performed several test fits with the PEXRAV model and a Gaussian line separately and we get almost identical results like with PEXMON, 


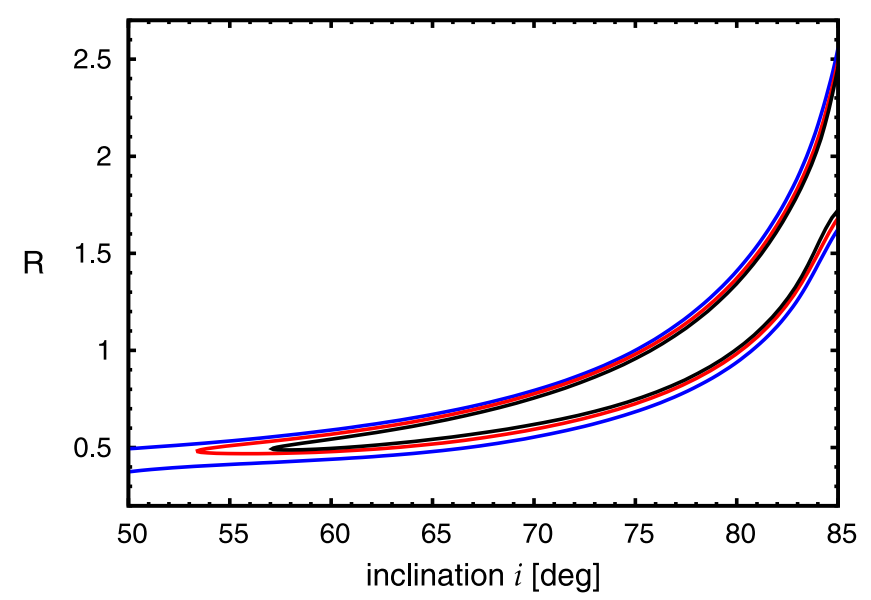

Fig. 9. Contour plot between the inclination angle and reflection parameter of the final model. The individual contours correspond to 68,90 , and $99 \%$ confidence levels.

i.e. favouring a high inclination angle measured relatively to the normal of the orbital plane.

However, it is necessary to stress that the inclination angle intervenes only as a projection effect in the PEXRAV or PEXMON model. The shape of the emission is independent of the emission angle and the total spectrum is averaged over all angles. As a result, both $R$ and $i$ affect only the normalisation and are therefore degenerated. This is apparent in Fig. 9 where the confidence levels of the reflection parameter $R$ and the inclination angle $i$ of the final model are shown. Illumination of the accretion disc by an isotropic source on top of it corresponds to the value $R=1$. The larger values of $R$ would suggest that the more observed radiation comes from reflection than from the direct component.

The resulting high inclination angle of the final model, which is also suggested for the case $R=1$, is likely a consequence of the low contrast between the Compton scattering hump and the primary continuum, the former being determined by the combination of strong Compton scattering and low photoelectric absorption cross-section. The lower inclination would imply the Compton hump to be more prominent given the observed equivalent width of the iron line.

This would imply two possible scenarios. First, the system could be oriented almost face-on but we see the reflection on the distant broad line region or the torus, which gives a complementary reflection angle. This is supported by the detection of broad lines in the optical spectrum (Piconcelli et al. 2006). It is also consistent with low inclination angle of the innermost accretion disc measured by the relativistic iron line model in the flat power-law scenario for the XMM-Newton spectrum (see Fig. 6). The second scenario assumes that the reflection occurs at the more distant parts of an accretion disc which is oriented almost edge-on relatively to the observer.

The edge-on disc hypothesis is not in a strong contradiction with the optical measurements. The standard unification scenario has been questioned in several sources, for a review see Bianchi et al. (2012) and references therein. The clumpiness of the absorber is supposed to be another important parameter in the proper classification and the type of AGN could not be entirely determined by the inclination angle but also by a probability of the absorption clouds intercepting the line of sight (Nenkova et al. 2002; Elitzur 2012). This is supported by a large variability of the absorber observed in several sources (see e.g. Risaliti et al. 2005). 4U 1344-60 has also revealed partially covering absorber with a significant change between the archival XMM-Newton and recent Suzaku observation, and can be thus another example advocating for an updated unification model.

Moreover, the inclination angle of the accretion disc and the host galaxy might be misaligned (see e.g. Guainazzi et al. 2011). An evident misalignment of the circum-nuclear matter with the radio jet, which is naturally believed to lie in the black hole rotation axis, was reported by Raban et al. (2009). Risaliti et al. (2011) analysed the distribution of the [O III] emission line equivalent width in order to constrain the inclination in a large sample of AGNs, and reported several sources with edge-on discs but not heavily absorbed by a circum-nuclear matter. $4 \mathrm{U}$ 1344-60 could belong to this kind of sources as well.

\section{Conclusions}

The X-ray continuum spectrum of the Seyfert galaxy $4 \mathrm{U} 1344-60$ is dominated by a power law with a standard value of the photon index $\Gamma \approx 1.7 \pm 0.1$. A narrow fluorescent line with equivalent width $\approx 100 \mathrm{eV}$ is an apparent feature in the XIS spectra. The width of the iron line is $\sigma=(0.08 \pm 0.02) \mathrm{keV}$, i.e. $\approx 9000 \mathrm{~km} \mathrm{~s}^{-1}$ of FWHM, suggesting its origin from a distant matter not affected by relativistic smearing. The outermost parts of an accretion disc, broad line region clouds or torus are possible candidates for the reflector. Its profile is, however, significantly narrower than in the archival XMM-Newton observation. The detected red-shifted iron line emission during the $X M M$-Newton observation was likely a temporary feature either due to locally enhanced emission or decreased ionisation in the innermost accretion flow.

The spectral shape has also changed between the XMM-Newton and Suzaku observation. We interpret this change as due to the presence of a partially covering absorber. The absorbed fraction of the primary radiation has decreased from around $45 \%$ to less than $10 \%$. The resulting model is then characterised by the same power law slope. It is also consistent with the flux measured (non-simultaneously) by the INTEGRAL and Swift satellite at high X-ray energies. The spectral variability makes 4U 1344-60 an interesting target for further examination within a monitoring programme by an X-ray satellite.

Acknowledgements. The work was supported from the grant ME09036 in the Czech Republic (V.K.). J.S. acknowledges discussion with Andy Pollock about the statistical processing of the data and with other colleagues attending Scientific Mondays at ESAC.

\section{Appendix A}

\section{A.1. Timing and spectral properties of Centaurus $B$ from ASCA to Suzaku}

Centaurus $\mathrm{B}$ is one of the nearest and brightest radio galaxies, at cosmological redshift $z=0.01215$ and flux density $\approx 250 \mathrm{Jy}$ at $408 \mathrm{MHz}$ (Jones et al. 2001). It is located only at $13^{\prime}$ from $4 \mathrm{U} 1344-60$ on the sky. The proximity of the two sources did not allow us to set an observing configuration in which we can exclude Centaurus B from the HXD detectors. Therefore, we required an accompanying $10 \mathrm{ks}$ observation targeted directly on Cen B to estimate its X-ray flux in HXD/PIN energy range. Here, we present our spectral results from this short Suzaku observation using the XIS detectors together with the analysis of its long-term variability. The data reduction procedure is the same as described in Sect. 2.

Historically, the flux of Centaurus B was found to be only a factor of a few smaller than 4U 1344-60. The observed flux 

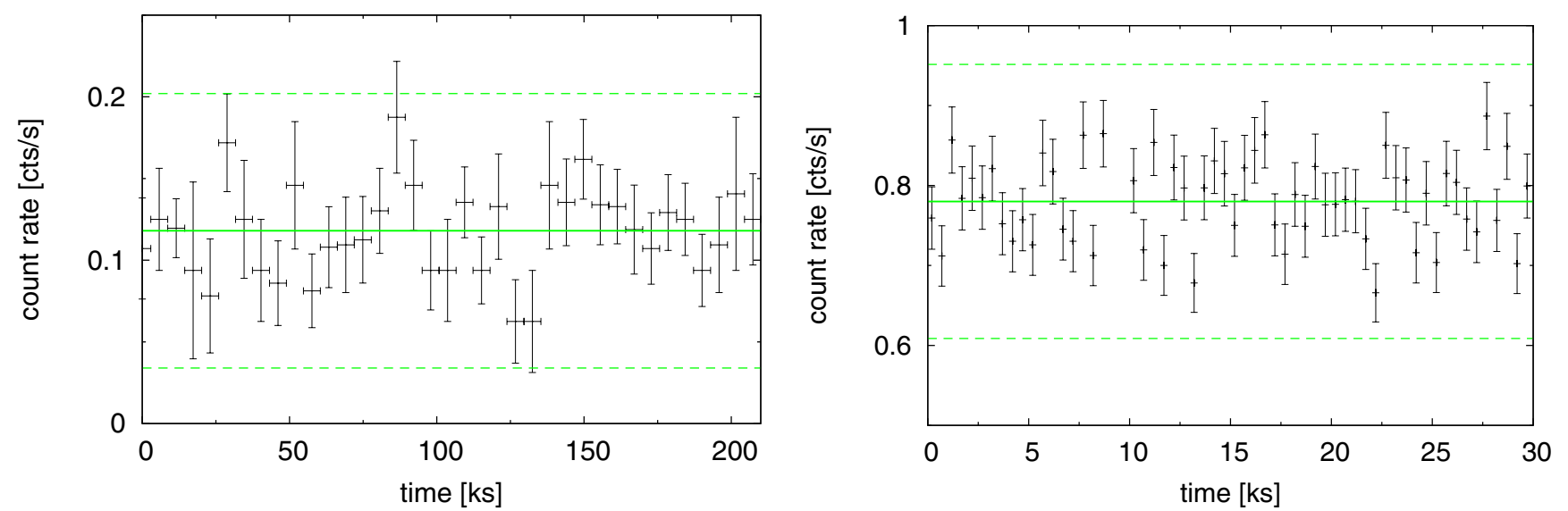

Fig. A.1. Light curve of Centaurus B. Left: ASCA (200 ks). Right: XMM-Newton (30 ks). Horizontal solid lines represent the average flux. Dashed lines show the $3 \sigma$ levels where $\sigma$ is the expected standard deviation due to Poisson noise.

Table A.1. Evolution of the X-ray flux and spectral slope of Centaurus B.

\begin{tabular}{lccc}
\hline \hline & ASCA & XMM-Newton & SUZAKU \\
\hline $2-10 \mathrm{keV} \mathrm{flux}$ & & & \\
{$\left[10^{-12} \mathrm{erg} \mathrm{cm}^{-2} \mathrm{~s}^{-1}\right]$} & $7.3 \pm 0.2^{a}$ & $5.6 \pm 0.2$ & $3.9 \pm 0.2$ \\
photon index & $1.64 \pm 0.07^{a}$ & $1.6 \pm 0.1$ & $1.6 \pm 0.2$ \\
column density & $1.76 \pm 0.11^{a}$ & $1.7 \pm 0.1$ & $1.7 \pm 0.3$ \\
\hline
\end{tabular}

Notes. ${ }^{(a)}$ Tashiro et al. (1998); ${ }^{(b)}$ including Galactic absorption $\left(N_{\mathrm{H}}=\right.$ $1.06 \times 10^{22} \mathrm{~cm}^{-2}$ ).

in the $2-10 \mathrm{keV}$ was $7.3 \pm 0.2 \times 10^{-12} \mathrm{erg} \mathrm{cm}^{-2} \mathrm{~s}^{-1}$ in the archival ASCA observation (Tashiro et al. 1998), and $5.6 \pm$ $0.2 \times 10^{-12} \mathrm{erg} \mathrm{cm}^{-2} \mathrm{~s}^{-1}$ in the XMM-Newton observation (see also Tashiro et al. 2005), i.e. around 6-7 times fainter than 4U 1344-60. The INTEGRAL satellite measured hard X-ray fluxes of $f_{20-40 \mathrm{keV}}=(0.76 \pm 0.08) \times 10^{-11} \mathrm{erg} \mathrm{cm}^{-2} \mathrm{~s}^{-1}$ and $f_{40-100 \mathrm{keV}}=(1.1 \pm 0.2) \times 10^{-11} \mathrm{erg} \mathrm{cm}^{-2} \mathrm{~s}^{-1}$ (Bird et al. 2010), around 4 times less than the $4 \mathrm{U}$ 1344-60 fluxes.

Figure A.1 shows X-ray light curves from the archival ASCA and $X M M-N e w t o n$ observations. The light curves look stationary with no trend in the flux. We fitted the light curves by polynomial fits, and we found that a constant function represents the best fit. Most of the apparent variability in the light curves is due to Poisson noise whose level is shown by the dashed lines in the plots. These results clearly suggest very low variability and, in particular, no trend in the average flux of Cen $B$ on $a \approx 100 \mathrm{ks}$ time-scale.

The 2-10 keV flux measured with the Suzaku/XIS detectors is $(3.9 \pm 0.2) \times 10^{-12} \mathrm{erg} \mathrm{cm}^{-2} \mathrm{~s}^{-1}$, which follows a continuous decrease of the flux since the ASCA observation (see Table A.1). The extrapolated $15-60 \mathrm{keV}$ flux is then approximately $8 \times 10^{-12} \mathrm{erg} \mathrm{cm}^{-2} \mathrm{~s}^{-1}$, which is one order of magnitude less than $4 \mathrm{U}$ 1344-60. Thus, in the hard X-ray regime, the flux of 4U 1344-60 clearly dominates.

Although the X-ray brightness of Cen B has decreased by a factor of two from the archival ASCA observation in 1995 to the recent Suzaku observation in 2011 the spectral shape has not changed (see Table A.1) and can be characterised by a simple power law with the photon index $\approx 1.6$ and local absorption with the column density $N_{\mathrm{H}} \approx 0.7 \times 10^{22} \mathrm{~cm}^{-2}$. The Suzaku/XIS spectrum of Centaurus B is shown in Fig. A.2. The underlying model is TBABS*ZTBABS*ZPOWERLW in the XSPEC notation with the best-fit values from the Table A.1. The statistical goodness of

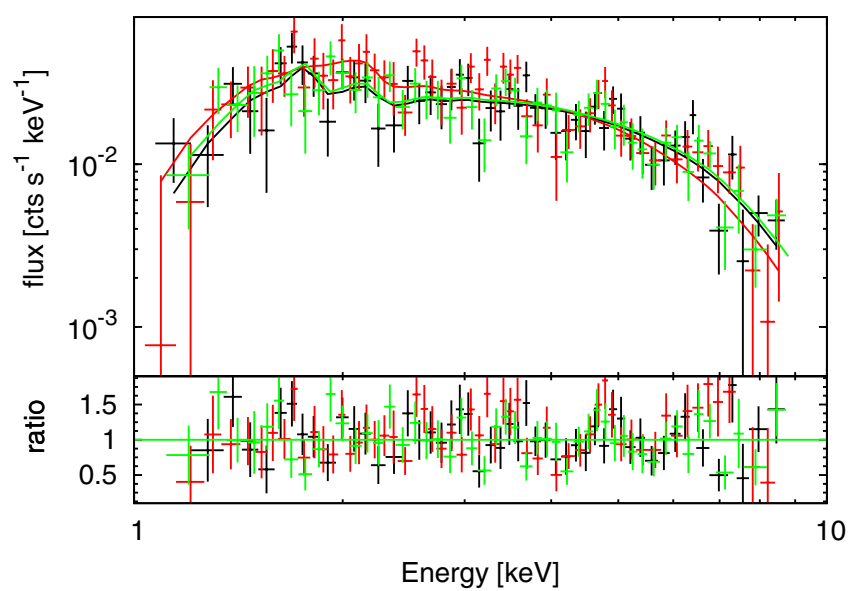

Fig. A.2. X-ray spectrum of Centaurus B observed with Suzaku XIS 0 (black), XIS 1 (red), XIS 3 (green).

the fit can be characterised by $C / v=177 / 162$ (or $\chi^{2} / v=$ $169 / 162 \approx 1.04$ ). Some residuals from the model are apparent in the spectrum but they are likely due to a less than perfect subtraction of the background, considering the relatively low flux of CenB (the fraction of the background flux is around 30\%).

\section{A.2. Estimate of the contamination of the $4 U$ 1344-60 HXD/PIN spectrum by Centaurus $B$}

Although the high energy flux of Centaurus B is one order of magnitude lower, we considered it for the proper crossnormalisation between XIS and HXD/PIN instruments. We performed a fit of HXD/PIN spectrum of 4U 1344-60 with a single and double power-law model. The second power-law component did not improve the fit at all. However, a slightly flatter photon index was preferred using the single power-law model. This would be an expectable influence of the contamination by a harder Centaurus $\mathrm{B}$ spectrum with the photon in$\operatorname{dex} \Gamma \approx 1.6 \pm 0.2$. Although the determined error of the photon index from the short Suzaku observation is rather high, the value of 1.6 has been consistently measured since the earliest ASCA observation (see Table A.1).

We used the best fit values obtained from the analysis of the XIS spectra of Centaurus B multiplied by a constant between 0 and 1 . We fitted the XIS and HXD/PIN spectra simultaneously with the final C-value 7847 with 7505 degrees of 
freedom, or $\chi_{v}^{2}=5839 / 5645$, respectively. This means only a marginal improvement with $\Delta C \approx 3$ (or $\Delta \chi \approx 4$, respectively) compared to the model without any contamination taken into account. The best-fit value for the constant is $c=0.1_{-0.1}^{+0.4}$. Given the fact that the flux of Centaurus $\mathrm{B}$ is one order of magnitude weaker than for $4 \mathrm{U} 1344-60$ the contamination is $5 \%$ at maximum.

\section{References}

Antonucci, R. 1993, ARA\&A, 31, 473

Bhayani, S., \& Nandra, K. 2011, MNRAS, 416, 629

Bianchi, S., Guainazzi, M., Matt, G., Fonseca Bonilla, N., \& Ponti, G. 2009, A\&A, 495, 421

Bianchi, S., Maiolino, R., \& Risaliti, G. 2012, Adv. Astron., 2012

Bianchi, S., Matt, G., Balestra, I., Guainazzi, M., \& Perola, G. C. 2004, A\&A, 422,65

Bird, A. J., Bazzano, A., Bassani, L., et al. 2010, ApJS, 186, 1

Brenneman, L. W., \& Reynolds, C. S. 2009, ApJ, 702, 1367

Cash, W. 1979, ApJ, 228, 939

Cusumano, G., La Parola, V., Segreto, A., et al. 2010, A\&A, 524, A64

Czerny, B., Różańska, A., Dovčiak, M., Karas, V., \& Dumont, A.-M. 2004, A\&A, 420, 1

de La Calle Pérez, I., Longinotti, A. L., Guainazzi, M., et al. 2010, A\&A, 524, A50

Dickey, J. M., \& Lockman, F. J. 1990, ARA\&A, 28, 215

Done, C., Madejski, G. M., \& Życki, P. T. 2000, ApJ, 536, 213

Dovčiak, M., Bianchi, S., Guainazzi, M., Karas, V., \& Matt, G. 2004a, MNRAS, 350,745

Dovčiak, M., Karas, V., \& Yaqoob, T. 2004b, ApJS, 153, 205

Elitzur, M. 2012, ApJ, 747, L33

Esin, A. A., McClintock, J. E., \& Narayan, R. 1997, ApJ, 489, 865

Fender, R. P., Belloni, T. M., \& Gallo, E. 2004, MNRAS, 355, 1105

Goosmann, R. W., Mouchet, M., Czerny, B., et al. 2007, A\&A, 475, 155

Guainazzi, M., Bianchi, S., \& Dovčiak, M. 2006, Astron. Nachr., 327, 1032

Guainazzi, M., Bianchi, S., de La Calle Pérez, I., Dovčiak, M., \& Longinotti, A. L. 2011, A\&A, 531, A131

Iwasawa, K., Miniutti, G., \& Fabian, A. C. 2004, MNRAS, 355, 1073

Jansen, F., Lumb, D., Altieri, B., et al. 2001, A\&A, 365, L1

Jiménez-Bailón, E., Piconcelli, E., Guainazzi, M., et al. 2005, A\&A, 435, 449
Jones, P. A., Lloyd, B. D., \& McAdam, W. B. 2001, MNRAS, 325, 817

Kalberla, P. M. W., Burton, W. B., Hartmann, D., et al. 2005, A\&A, 440, 775

Lubiński, P., \& Zdziarski, A. A. 2001, MNRAS, 323, L37

Magdziarz, P., \& Zdziarski, A. A. 1995, MNRAS, 273, 837

Maiolino, R., \& Rieke, G. H. 1995, ApJ, 454, 95

Markowitz, A. G., \& Reeves, J. N. 2009, ApJ, 705, 496

Matt, G. 2000, A\&A, 355, L31

Matt, G., Fabian, A. C., \& Ross, R. R. 1993, MNRAS, 262, 179

Matt, G., Porquet, D., Bianchi, S., et al. 2005, A\&A, 435, 857

Miller, L., Turner, T. J., \& Reeves, J. N. 2008, A\&A, 483, 437

Mitsuda, K., Bautz, M., Inoue, H., et al. 2007, PASJ, 59, 1

Murphy, K. D., \& Yaqoob, T. 2009, MNRAS, 397, 1549

Nandra, K., O’Neill, P. M., George, I. M., \& Reeves, J. N. 2007, MNRAS, 382, 194

Nenkova, M., Ivezić, Ž., \& Elitzur, M. 2002, ApJ, 570, L9

Nousek, J. A., \& Shue, D. R. 1989, ApJ, 342, 1207

Panessa, F., Bassani, L., de Rosa, A., et al. 2008, A\&A, 483, 151

Peterson, B. M. 2006, in Physics of Active Galactic Nuclei at all Scales, ed. D. Alloin, Lecture Notes in Physics (Berlin: Springer Verlag), 693, 77

Piconcelli, E., Sánchez-Portal, M., Guainazzi, M., et al. 2006, A\&A, 453, 839

Raban, D., Jaffe, W., Röttgering, H., Meisenheimer, K., \& Tristram, K. R. W. 2009, MNRAS, 394, 1325

Read, A. M., Rosen, S. R., Saxton, R. D., \& Ramirez, J. 2011, A\&A, 534, A34

Reynolds, C. S., \& Fabian, A. C. 2008, ApJ, 675, 1048

Reynolds, C. S., \& Nowak, M. A. 2003, Phys. Rep., 377, 389

Risaliti, G., Elvis, M., Fabbiano, G., Baldi, A., \& Zezas, A. 2005, ApJ, 623, L93

Risaliti, G., Salvati, M., \& Marconi, A. 2011, MNRAS, 411, 2223

Sochora, V., Karas, V., Svoboda, J., \& Dovčiak, M. 2011, MNRAS, 418, 276

Svoboda, J., Guainazzi, M., \& Karas, V. 2010, A\&A, 512, A62

Svoboda, J., Dovčiak, M., Goosmann, R. W., et al. 2012, A\&A, 545, A106

Tashiro, M., Kaneda, H., Makishima, K., et al. 1998, ApJ, 499, 713

Tashiro, M., Isobe, N., Suzuki, M., et al. 2005, in X-Ray and Radio Connections, eds. L. O. Sjouwerman, \& K. K. Dyer

Turner, T. J., Mushotzky, R. F., Yaqoob, T., et al. 2002, ApJ, 574, L123

Turner, T. J., Miller, L., Kraemer, S. B., \& Reeves, J. N. 2011, ApJ, 733, 48

Uzdensky, D. A., \& Goodman, J. 2008, ApJ, 682, 608

Vasudevan, R. V., Fabian, A. C., Gandhi, P., Winter, L. M., \& Mushotzky, R. F. 2010, MNRAS, 402, 1081

Wilms, J., Allen, A., \& McCray, R. 2000, ApJ, 542, 914

Yaqoob, T. 2012, MNRAS, 423, 3360 